\title{
KIT genetic alterations in anorectal melanomas
}

\author{
Raffaella Santi, ${ }^{1}$ Lisa Simi, ${ }^{2}$ Rossella Fucci, ${ }^{2}$ Milena Paglierani, ${ }^{1}$ Monica Pepi, \\ Pamela Pinzani, ${ }^{2}$ Barbara Merelli, ${ }^{3}$ Marco Santucci, ${ }^{1}$ Gerardo Botti, ${ }^{4}$ Carmelo Urso, ${ }^{5}$ \\ Daniela Massi ${ }^{1}$
}

- Additional material is published online only. To view please visit the journal online (http://dx.doi.org/10.1136/ jclinpath-2014-202572).

${ }^{1}$ Division of Pathological Anatomy, Department of Surgery and Translational Medicine, University of Florence, Florence, Italy ${ }^{2}$ Clinical Biochemistry Unit, Department of Biomedical, Experimental and Clinical Sciences, University of Florence, Florence, Italy ${ }^{3}$ Department of Oncology and Haematology, Papa Giovanni XXIII Hospital, Bergamo, Italy ${ }^{4}$ Department of Pathology, National Cancer Institute "Fondazione G. Pascale", Naples, Italy

${ }^{5}$ Dermatopathology Section, S.M. Annunziata Hospital, ASL 10, Florence, Italy

\section{Correspondence to} Raffaella Santi, Division of Pathological Anatomy, Department of Surgery and Translational Medicine, University of Florence, Largo Brambilla, 3, Florence I-50134, Italy;

raffaella.santi@yahoo.it

Received 23 July 2014 Revised 13 October 2014 Accepted 25 October 2014

\section{ABSTRACT}

Background Mucosal melanomas (MM) represent a heterogeneous tumour population that exhibits sitespecific molecular profiles.

Aims In a multicentre retrospective study, we investigated KIT aberrations in primary anorectal (AR) melanomas compared with melanoma metastatic to the gastrointestinal (GI) tract.

Methods Primary AR MM $(n=31)$ and GI metastatic melanoma $(n=27)$ were studied for KIT mutations on exons $11,13,17$ and 18 by high-resolution melting analysis, direct sequencing and c-KIT expression by immunohistochemistry. Selected cases were also investigated for increased KIT gene copy number by fluorescent in situ hybridisation.

Results Functional KIT mutations were demonstrated in $11 / 31(35.5 \%)$ of AR melanomas and in $1 / 26(3.8 \%)$ of $\mathrm{Gl}$ melanoma metastases $(p=0.004)$. A significant difference emerged between primary and metastatic MM with regards to KIT-positive immunostaining $(p=0.002)$. Immunohistochemical c-KIT protein overexpression did not correlate with KIT mutational status. Increased KIT copy number was demonstrated in 5/20 AR primary cases. Conclusions The rate of functional mutations in KIT is significantly higher in AR MM than in GI metastatic melanoma. KIT protein overexpression does not correlate with KIT mutations and cannot be used for screening purposes. Recognising the molecular heterogeneity of MM helps to identify patients who require a different therapeutic approach.

\section{INTRODUCTION}

Melanomas of the gastrointestinal (GI) tract are rare neoplasms, often representing metastatic disease located in the small bowel. ${ }^{1}$ In autopsy series, $43.5 \%$ $60 \%$ of patients who die of cutaneous melanoma have GI metastases. ${ }^{2-4}$ Nevertheless, mainly being asymptomatic, $<5 \%$ of metastases to the GI tract are clinically diagnosed during the patient's lifetime. ${ }^{1}$ Primary mucosal melanomas (MM) account for approximately $1.3 \%$ of all melanomas. ${ }^{5}$ Anorectal (AR) melanomas constitute $23.8 \%$ of primary MM, whereas head and neck region, the female genital tract and the urinary tract represent $55.4 \%, 18 \%$ and $2.8 \%$ of cases, respectively. ${ }^{5}$ Although rarely, $\mathrm{MM}$ in the GI tract have been found in the oesophagus, stomach, small and large bowel. ${ }^{67}$

GI melanomas are associated with a severe prognosis regardless of the therapy employed. ${ }^{6}$ For current and more effective personalised therapies, incorporation of genetic signatures into the morphological classification of melanoma is mandatory. ${ }^{89}$ In recent years, the use of kinase inhibitors in patients with melanoma with documented KIT mutations has shown promising results. ${ }^{10-16}$

The reported frequency of KIT mutations in MM varies between 5.4\% and 38\% (mean 17.6\%; median 17\%). ${ }^{17-32}$ Frequent association between KIT mutations and overexpression of c-KIT protein has been reported, ${ }^{17} 1820-22252629$ however, data are still conflicting. ${ }^{15} 19$ 30-33

To identify the molecular signature of primary AR melanomas, we investigated KIT aberrations by evaluating KIT mutational status and c-KIT protein immunohistochemical expression in a series of 31 primary AR melanomas compared with 27 cases of cutaneous melanoma metastatic to the GI tract. Selected AR cases were also tested for KIT gene copy number abnormalities by fluorescent in situ hybridisation (FISH) analysis.

\section{MATERIALS AND METHODS \\ Tumour tissue samples}

We searched the patient database at the Pathological Anatomy Units belonging to the Tumour Institute of Tuscany (ITT) network (Italy), at the Department of Pathology, National Cancer Institute 'Fondazione G. Pascale' of Naples (Italy) and at the Department of Oncology and Haematology, Papa Giovanni XXIII Hospital of Bergamo (Italy), for cases diagnosed between 1991 and 2013 as primary or metastatic melanoma involving the GI tract. Institutional Review Board was informed and consented in the study. Histological sections were reviewed by two pathologists independently to confirm the diagnosis. In each case, a representative formalin-fixed, paraffin-embedded block was selected and used for immunohistochemistry as well as for molecular studies. Since exons 11, 13, 17 and 18 are described as common mutation sites of KIT in melanomas, ${ }^{17} 22$ the study focused on the detection of somatic mutations in these exons. FISH analysis was performed in seven cases to exclude the presence of $t(12 ; 22)$ (q13; q12) translocation (r/o soft parts/clear cell sarcoma). FISH was also employed for KIT amplification analyses and high-resolution melting analysis (HRMA) and Sanger sequencing were used for mutational testing. For detailed description of immunohistochemistry, FISH and gene mutation analysis, see online supplementary file 1 .

\section{Evaluation and statistics}

Immunohistochemical stains were evaluated for the percentage of labelled cells and the intensity of immunoreactivity according to the grading criteria suggested by Alexis and coauthors. ${ }^{34}$ The amount of immunopositive cells was reported as follows: 0 , no staining or weak staining in individual cells; $1+$, 


\section{Original article}

spotty weak staining of groups of cells; $2+$, diffuse weak staining or moderate staining of up to $50 \%$ of cells; $3+$, moderate staining of $>50 \%$ to $75 \%$ of cells; $4+$, moderate to strong staining of $>75 \%$ of cells. For statistical purposes, we then divided cases into two groups based on KIT immunohistochemical expression: melanomas overexpressing KIT (scored as $3+$ or $4+$ ) and melanomas with low/absent KIT expression (tumours scored as $0 / 1+/ 2+)$.

We accessed the Sanger COSMIC (Catalogue of Somatic Mutations in Cancer) databank (http://www.sanger.ac.uk/ genetics/CGP/cosmic/) in order to compare previously reported KIT mutations with our results. We have taken into account only KIT functional mutations that are known/expected to affect the protein phenotype.

The software SPSS for Windows V.17.0 (SPSS, Chicago, Illinois, USA) was used for statistical analysis. A two-tailed Pearson's $\chi^{2}$ test was performed, and a value of $\mathrm{p} \leq 0.05$ was considered statistically significant.

\section{RESULTS}

\section{Patient characteristics and follow-up data}

Among patients affected by primary AR melanomas $(n=31)$, age ranged from 56 to 89 years (median 73 years) and included $64.5 \%$ (20/31) women and $35.5 \%$ (11/31) men. Mean follow-up period was 18.3 months (data were available for 28/ 31 patients). Anatomical sites of GI metastases $(n=27)$ included small bowel $(n=15)$, large bowel $(n=5)$, stomach $(n=3)$ and oesophagus $(n=1)$. Three patients presented with multiple lesions, comprising different and/or distant GI organs and the omentum. Age ranged from 26 to 82 years (median 63 years) and included $66.7 \%(18 / 27)$ men and $33.3 \%$ (9/27) women. Mean follow-up period was 23.5 months (data were available in 21/27 cases).

\section{Histological features of AR melanomas}

AR melanomas generally presented as exophytic, diffusely ulcerated lesions. Median tumour size was $3.3 \mathrm{~cm}$ (range $0.4-7 \mathrm{~cm}$ ). Sixteen AR melanomas $(16 / 31 ; 51.6 \%)$ were amelanotic; melanin pigment, at least focally, was present in the remaining cases. Epithelioid melanomas represented the most common subtype $(17 / 31 ; 54.8 \%)$, whereas epithelioid and spindle $(n=11$; $35.5 \%)$ and spindle $(n=3 ; 9.7 \%)$ melanomas were less frequently encountered. Primary AR melanomas were all invasive and Breslow thickness ranged from 5 to $15 \mathrm{~mm}$ (mean $10.5 \mathrm{~mm}$ ) with a mitotic index varying from 6 to 30 mitoses $/ \mathrm{mm}^{2}$ (mean 11.9 mitoses $/ \mathrm{mm}^{2}$ ). Of the nine surgical resection specimens, five were confined to the visceral wall, whereas extension to the perivisceral fat was observed in the remaining cases.

\section{Immunohistochemical results}

Overall, c-KIT overexpression (score $3+/ 4+$ ) was observed in $18 / 31(58.1 \%)$ primary AR melanomas and in 5/27 (18.5\%) metastatic cases. Absent or low c-KIT expression (score 0, 1+, $2+$ ) was demonstrated in 13/31 (41.9\%) primary tumours and in $22 / 27(81.5 \%)$ metastatic cases (figure 1). This difference was statistically significant $(p=0.002)$.

\section{Mutational analysis results}

Mutational analysis of the KIT gene was performed in all primary AR melanomas and in 26/27 metastatic cases.

In AR melanomas, four cases were found to have mutations on exon 11: the L576P mutation $(n=3)$ and a three-nucleotide deletion p.Y578_D579del $(n=1)$, previously reported in the Sanger COSMIC databank (figure 2). Seven cases showed mutations on exon 17: the p.Y823D mutation $(n=6)$ and the silent p.R804R mutation $(n=1)$. On exon 13 , the p.K642R mutation was identified in one case.

Among metastatic melanomas, one of three gastric cases harboured the p.Y823D mutation on exon 17, whereas in a small bowel MM a silent mutation (p.V555V) on exon 11 was detected. No genetic alterations were detected on exon 13 .

Exon 18 resulted wild-type in all $57 \mathrm{MM}$ cases.

A statistically significant difference emerged in the frequency of functional KIT mutations (missense and frameshift mutations) between primary and metastatic cases $(11 / 31,35.5 \%$ vs $1 / 26$, $3.8 \%, \mathrm{p}=0.004)$.

\section{Genotype/phenotype correlation}

Among the 22 samples with immunohistochemical score $3+$ or $4+, 45.5 \%(10 / 22)$ harboured functional mutations, whereas $54.5 \%(12 / 22)$ were KIT wild-type. In contrast, among the 35 samples with immunohistochemical score $0 / 1+/ 2+$, only $5.7 \%$ $(2 / 35)$ harboured the p.Y823D mutation on exon 17 , whereas 33/35 (94.3\%) were KIT wild-type.

\section{KIT copy number increase}

Extra copies of KIT were found in 5/20 AR melanomas (25\%). These tumours showed c-KIT overexpression, score $3+(n=1)$ and $4+(n=4)$. At sequencing analysis, four cases were KIT wildtype, whereas one also harboured the p.Y823D mutation (figure 3).

\section{DISCUSSION}

The most striking result of the present study is that the frequency of functional KIT gene mutations is significantly higher in AR MM than melanomas metastatic to the GI tract $35.5 \%$ vs $3.8 \%, \mathrm{p}=0.004)$. Previous studies reported a range of KIT mutations in $\mathrm{MM}$ of the GI tract between $5.4 \%$ and $38 \%$
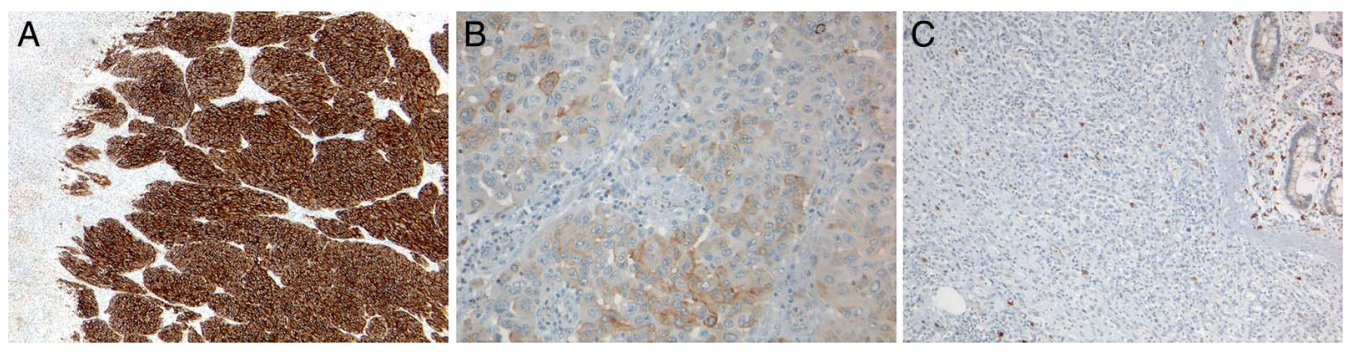

Figure 1 (A) Diffuse, strong immunohistochemical expression of c-KIT in a case of primary anorectal melanoma (original magnification $\times 10$ ); (B) a case of cutaneous melanoma metastatic to the small bowel showing weak C-KIT immunostaining (original magnification $\times 20$ ); (C) lack of c-KIT-positive cells in a metastatic melanoma of the small bowel (original magnification $\times 10$ ). 

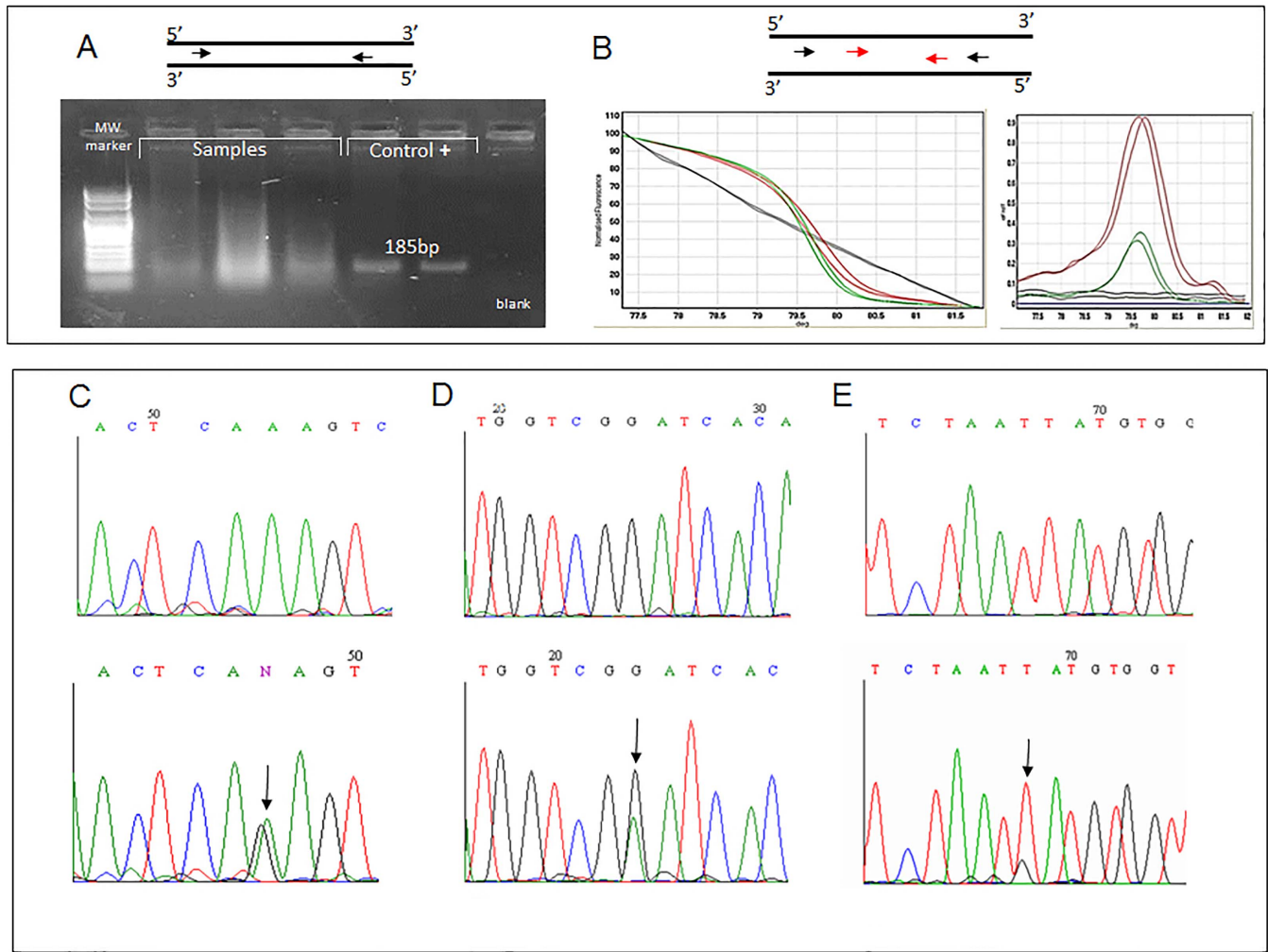

Figure 2 Upper row: schematic representation of the preAmp-high-resolution melting analysis (HRMA) methodological approach. (A) Absence of specific amplification on KIT exon 17 after a direct PCR; (B) specific amplification on KIT exon 17 demonstrated after the preAmp-HRMA protocol, which includes a second amplification with internal primers (tested sample: red line; positive control: green line; direct PCR: black line). Lower row: electropherograms of mutated samples. (C) K642R mutation detected in the exon 13, consequently to an AAA $>A G A$ substitution; (D) CGG $>C G A$ substitution responsible of the R804R silent mutation; (E) the p.Y823D mutation caused by a TAT>GAT substitution.

(median 17\%). ${ }^{17-32}$ Regarding AR MM the reported frequency varied between $0 \%$ and $67 \%$ (median 15\%). ${ }^{18} 202324263032$

In our series, the frequency of mutated samples was higher than the median frequency previously acknowledged. The wide range of KIT frequencies so far reported in AR melanomas may reflect different inclusion criteria and small sample size bias, with number of cases varying between 2 and 40 cases (mean 17.4; median 21). ${ }^{1820232426} 3032$

Differentiating primary from metastatic GI melanomas can be challenging. ${ }^{6} 7$ Suggestive features of the primary nature of GI melanoma include lack of concurrent or previous removal of a melanoma or atypical melanotic lesion from the skin, lack of other organ involvement and in situ changes in the overlying or adjacent GI epithelium. ${ }^{35}$ Differential diagnosis of MM includes clear cell sarcoma and GIST. Clinical presentation, appropriate immunohistochemistry and molecular biology tests should be considered to avoid diagnostic misinterpretation with relevant therapeutic implications.

To the best of our knowledge, this is one of the largest series of primary and metastatic GI tract melanomas so far reported.

Among the 11 AR MM, three tumours harboured the L576P mutation on exon 11. These patients were treated with imatinib and are still alive and with response after 10, 12 and 14 months, respectively. In our series, seven patients harboured the p.Y823D mutation, which appears to determine a loop alteration in the c-KIT protein that, in turn, may predict resistance to imatinib in GIST. ${ }^{36}$ No similar data have been reported in in vitro or in in vivo melanoma models. Recent data suggest that flumatinib, an inhibitor of BCR-ABL/PDGFR/KIT, has superior efficacy compared with imatinib or sunitinib against GIST cell
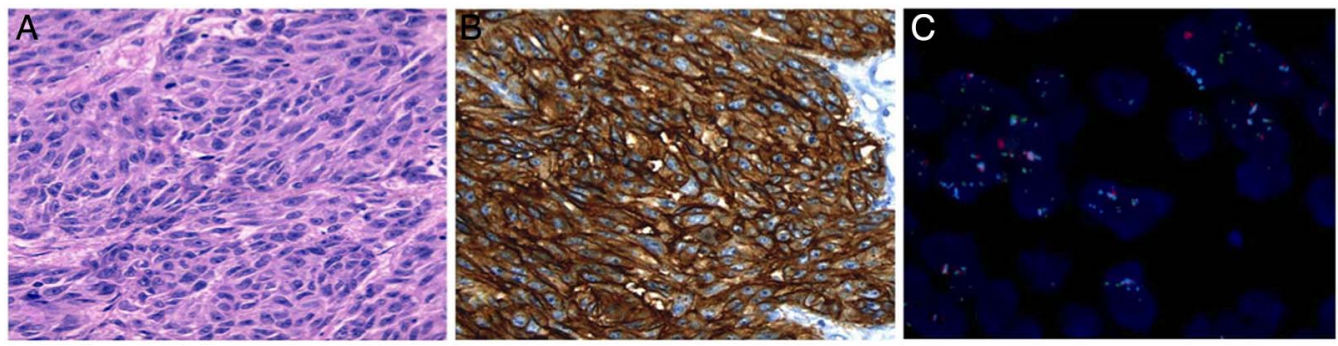

Figure 3 (A) A case of anorectal melanoma resulted wild-type for KIT (original magnification $\times 20$ ); (B) strong and diffuse c-KIT immunohistochemical staining, original magnification $40 x$; (C) fluorescent in situ hybridisation analysis for KIT showing an increase in fused signals for probes to 4q12 (LSI 4q12 Tri-Colour Rearrangement Probe-Vysis-Abbott Molecular). 
lines with the secondary mutation p.Y823D. ${ }^{36}$ Since KIT inhibitors are target-oriented agents, we could hypothesise that drugs overcoming loop mutations may be an effective therapy in MM with KIT mutations conferring imatinib resistance. ${ }^{16} 3738$

Overall, 12/45 (26.7\%) KIT wild-type tumours showed a high percentage of c-KIT strongly positive cells (scores $3+, 4+$ ) at immunohistochemical analysis. Among these, four cases showed an increased KIT copy number at FISH analysis. One AR melanoma with increased KIT copy number also presented the p. Y823D mutation on exon 17. KIT increased copy number and amplification have been reported in primary MM, either with or without mutations. ${ }^{17-19} 26-283031$ It has been suggested that KIT gene amplification is an unusual event in AR melanomas, although modest increase in KIT copy number can be seen in up to one-third of cases. ${ }^{18}{ }^{19}$ Increased KIT copy number, co-overexpression of cyclin-dependent kinase 4 and KIT, epigenetic mechanisms and autocrine/paracrine stimulation of KIT receptor have all been proposed to explain c-KIT protein expression in KIT wild-type cases. ${ }^{17} 22 \quad 28{ }^{39-41}$ However, KIT wild-type amplified tumours do not appear to be sensitive to tyrosine kinase inhibitors. ${ }^{37} 42$

Overall, our results suggest that immunohistochemical c-KIT overexpression does not correlate with KIT functional mutation. Although KIT mutations rarely occur in the absence of c-KIT protein expression, ${ }^{18}{ }^{22}$ CD117-negative staining cannot reliably rule out the presence of the mutation. ${ }^{15}$ 30-33

In summary, our data suggest that the incidence of activating KIT mutations is higher in AR MM than in melanoma metastasising to the GI tract. KIT-mutated MM are susceptible for therapy with specific kinase inhibitors and the recognition of the molecular heterogeneity of these tumours may help to identify patients requiring a different therapeutic approach.

\section{Take home messages}

- A significant number of anorectal (AR) mucosal melanomas (MM) harbour KIT activating mutations.

- Immunohistochemical c-KIT overexpression does not correlate with the presence of $K I T$ activating mutations.

- In AR MM, the spectrum of KIT genetic aberrations is wide and specific KIT mutations may have different sensitivity to c-KIT inhibition.

Acknowledgements This study was financially supported by fundings from Fondazione Cassa di Risparmio di Pistoia e Pescia (Pistoia, Italy). We would like to thank the following members of the Tumour Institute of Tuscany (ITT) network (Italy): Paola Apicella, Mauro Biancalani, Camilla Eva Comin, Morena Doria, Augusto Giannini, Roberto Incensati, Stefania Innocenti, Vincenza Maio, Luca Messerini, Clelia Miracco, Francesco Mirri, Luca Novelli, Loretta Presenti, Lavinia Pugliese, Armando Rossi, Carla Vindigni and Federica Zolfanelli.

Contributors According to the definition given by the International Committee of Medical Journal Editors (ICMJE), RS, LS, RF, MP, MP, PP, BM, MS, GB, CU and DM qualify for authorship based on making one or more of the substantial contributions to the intellectual content: conception and design, acquisition of data, and analysis and interpretation of data. Furthermore, they have participated in the drafting of the manuscript and critical revision of the manuscript for important intellectual content. All authors have seen and approved the final version of the manuscript before submission.

Funding Fondazione Cassa di Risparmio di Pistoia e Pescia.

Competing interests None.

Ethics approval The Institutional Review Board at each institution.

Provenance and peer review Not commissioned; externally peer reviewed.

\section{REFERENCES}

1 Lens $M$, Bataille V, Krivokapic Z. Melanoma of the small intestine. Lancet Oncol 2009;10:516-21.

2 Patel JK, Didolkar MS, Pickren JW, et al. Metastatic pattern of malignant melanoma. A study of 216 autopsy cases. Am J Surg 1978;135:807-10.

3 de la Monte SM, Moore GW, Hutchins GM. Patterned distribution of metastases from malignant melanoma in humans. Cancer Res 1983;43:3427-33.

4 Ihde JK, Coit DG. Melanoma metastatic to stomach, small bowel, or colon. Am J Surg 1991;162:208-11.

5 Chang AE, Karnell LH, Menck HR. The National Cancer Data Base report on cutaneous and noncutaneous melanoma: a summary of 84,836 cases from the past decade. The American College of Surgeons Commission on Cancer and the American Cancer Society. Cancer 1998;83:1664-78.

6 Manouras A, Genetzakis M, Lagoudianakis E, et al. Malignant gastrointestinal melanomas of unknown origin: should it be considered primary? World $J$ Gastroenterol 2007;13:4027-9.

7 Patrick RJ, Fenske NA, Messina JL. Primary mucosal melanoma. J Am Acad Dermatol 2007;56:828-34.

8 Romano E, Schwartz GK, Chapman PB, et al. Treatment implications of the emerging molecular classification system for melanoma. Lancet Oncol 2011;12:913-22.

9 Scolyer RA, Long GV, Thompson JF. Evolving concepts in melanoma classification and their relevance to multidisciplinary melanoma patient care. Mol Oncol 2011;5:124-36.

10 Quintás-Cardama A, Lazar AJ, Woodman SE, et al. Complete response of stage IV anal mucosal melanoma expressing KIT Val560Asp to the multikinase inhibitor sorafenib. Nat Clin Pract Oncol 2008;5:737-40.

11 Hodi FS, Friedlander $\mathrm{P}$, Corless $\mathrm{CL}$, et al. Major response to imatinib mesylate in KIT-mutated melanoma. J Clin Oncol 2008;26:2046-51.

12 Lutzky J, Bauer J, Bastian BC. Dose-dependent, complete response to imatinib of a metastatic mucosal melanoma with a K642E KIT mutation. Pigment Cell Melanoma Res 2008;21:492-3.

13 Woodman SE, Trent JC, Stemke-Hale K, et al. Activity of dasatinib against L576P KIT mutant melanoma: molecular, cellular, and clinical correlates. Mol Cancer Ther 2009;8:2079-85.

14 Satzger I, Küttler U, Völker B, et al. Anal mucosal melanoma with KIT-activating mutation and response to imatinib therapy-case report and review of the literature. Dermatology 2010;220:77-81.

15 Garrido MC, Bastian BC. KIT as a therapeutic target in melanoma. J Invest Dermatol 2010;130:20-7.

16 Carvajal RD, Antonescu CR, Wolchok JD, et al. KIT as a therapeutic target in metastatic melanoma. JAMA 2011;305:2327-34.

17 Curtin JA, Busam K, Pinkel D, et al. Somatic activation of KIT in distinct subtypes of melanoma. J Clin Oncol 2006;24:4340-6.

18 Antonescu CR, Busam KJ, Francone TD, et al. L576P KIT mutation in anal melanomas correlates with KIT protein expression and is sensitive to specific kinase inhibition. Int J Cancer 2007;121:257-64.

19 Beadling C, Jacobson-Dunlop E, Hodi FS, et al. KIT gene mutations and copy number in melanoma subtypes. Clin Cancer Res 2008;14:6821-8.

20 Satzger I, Schaefer T, Kuettler U, et al. Analysis of C-KIT expression and KIT gene mutation in human mucosal melanomas. Br J Cancer 2008;99:2065-9.

21 Rivera RS, Nagatsuka H, Gunduz M, et al. C-kit protein expression correlated with activating mutations in KIT gene in oral mucosal melanoma. Virchows Arch 2008;452:27-32.

22 Torres-Cabala CA, Wang WL, Trent J, et al. Correlation between KIT expression and KIT mutation in melanoma: a study of 173 cases with emphasis on the acral-lentiginous/mucosal type. Mod Pathol 2009;22:1446-56.

23 Handolias D, Hamilton AL, Salemi R, et al. Clinical responses observed with imatinib or sorafenib in melanoma patients expressing mutations in KIT. Br J Cancer 2010;102:1219-23.

24 Abysheva SN, lyevleva AG, Efimova NV, et al. KIT mutations in Russian patients with mucosal melanoma. Melanoma Res 2011;21:555-9.

25 Langer $\mathrm{R}$, Becker $\mathrm{K}$, Feith $\mathrm{M}$, et al. Genetic aberrations in primary esophageal melanomas: molecular analysis of c-KIT, PDGFR, KRAS, NRAS and BRAF in a series of 10 cases. Mod Pathol 2011;24:495-501.

26 Omholt K, Grafström E, Kanter-Lewensohn L, et al. KIT pathway alterations in mucosal melanomas of the vulva and other sites. Clin Cancer Res 2011;17:3933-42.

27 Yun J, Lee J, Jang J, et al. KIT amplification and gene mutations in acral/mucosal melanoma in Korea. APMIS 2011;119:330-5.

28 Kong Y, Si L, Zhu Y, et al. Large-scale analysis of KIT aberrations in Chinese patients with melanoma. Clin Cancer Res 2011;17:1684-91.

29 Abu-Abed S, Pennell N, Petrella T, et al. KIT gene mutations and patterns of protein expression in mucosal and acral melanoma. J Cutan Med Surg 2012;16:135-42.

30 Ni S, Huang D, Chen $X$, et al. C-kit gene mutation and CD117 expression in human anorectal melanomas. Hum Pathol 2012;43:801-7.

31 Minor DR, Kashani-Sabet M, Garrido M, et al. Sunitinib therapy for melanoma patients with KIT mutations. Clin Cancer Res 2012;18:1457-63. 
32 Schoenewolf NL, Bull C, Belloni B, et al. Sinonasal, genital and acrolentiginous melanomas show distinct characteristics of KIT expression and mutations. Eur $J$ Cancer 2012;48:1842-52

33 Park E, Yang S, Emley A, et al. Lack of correlation between immunohistochemical expression of CKIT and KIT mutations in atypical acral nevi. Am J Dermatopathol 2012:34:41-6.

34 Alexis JB, Martinez AE, Lutzky J. An immunohistochemical evaluation of c-kit (CD-117) expression in malignant melanoma, and results of imatinib mesylate (Gleevec) therapy in three patients. Melanoma Res 2005;15:283-5.

35 Blecker D, Abraham S, Furth EE, et al. Melanoma in the gastrointestinal tract. Am J Gastroenterol 1999;94:3427-33.

36 Zhao J, Quan $\mathrm{H}$, Xu Y, et al. Flumatinib, a selective inhibitor of BCR-ABL/PDGFR/ KIT, effectively overcomes drug resistance of certain KIT mutants. Cancer Sci 2014;105:117-25.
37 Hodi FS, Corless CL, Giobbie-Hurder A, et al. Imatinib for melanomas harboring mutationally activated or amplified KIT arising on mucosal, acral, and chronically sun-damaged skin. J Clin Oncol 2013;31:3182-90.

38 Guo J, Si L, Kong Y, et al. Phase II, open-label, single-arm trial of imatinib mesylate in patients with metastatic melanoma harboring c-Kit mutation or amplification. J Clin Oncol 2011;29:2904-9.

39 Lefevre G, Glotin AL, Calipel A, et al. Roles of stem cell factor/c-Kit and effects of Glivec/STI571 in human uveal melanoma cell tumorigenesis. J Biol Chem 2004;279:31769-79.

40 Smalley KS, Contractor R, Nguyen TK, et al. Identification of a novel subgroup of melanomas with KIT/yclin-dependent kinase-4 overexpression. Cancer Res 2008;68:5743-52.

41 Ashida A, Takata M, Murata H, et al. Pathological activation of KIT in metastatic tumors of acral and mucosal melanomas. Int I Cancer 2009;124:862-8.

42 Carvajal RD. Another option in our KIT of effective therapies for advanced melanoma. J Clin Oncol 2013;31:3173-5. 


\section{KIT genetic alterations in anorectal melanomas}

Raffaella Santi, Lisa Simi, Rossella Fucci, Milena Paglierani, Monica Pepi, Pamela Pinzani, Barbara Merelli, Marco Santucci, Gerardo Botti, Carmelo Urso and Daniela Massi

J Clin Pathol published online November 14, 2014

Updated information and services can be found at:

http://jcp.bmj.com/content/early/2014/11/14/jclinpath-2014-202572

Supplementary Material

References

Email alerting service
Supplementary material can be found at: http://jcp.bmj.com/content/suppl/2014/11/12/jclinpath-2014-202572.D C1.html

http://jcp.bmj.com/content/suppl/2014/11/12/jclinpath-2014-202572.D C2.html

These include:

This article cites 42 articles, 13 of which you can access for free at: http://jcp.bmj.com/content/early/2014/11/14/jclinpath-2014-202572 \#BIBL

Receive free email alerts when new articles cite this article. Sign up in the box at the top right corner of the online article.

\section{Notes}

To request permissions go to:

http://group.bmj.com/group/rights-licensing/permissions

To order reprints go to:

http://journals.bmj.com/cgi/reprintform

To subscribe to BMJ go to:

http://group.bmj.com/subscribe/ 